\title{
Morbi-mortalidad Perinatal
}

\author{
Dres.: Alfonso Jubiz Hasbun Juan L. Londoño Fernández
}

Asoc. Antioqueña de Obstetricia y Ginecología - Fundc. Hosp. San Vicente de Paul

\section{INTRODUCCION}

En el pasado Congreso Nacional de Obstetricia y Ginecología, realizado en la ciudad de Cartagena en diciembre de 1981 , se presentó a consideración de los especialistas un modelo de historia clínica perinatal, el cual fue diseñado en el CLAP y probado en varias maternidades de Latinoamérica.

Por dificultades en la aplicación del programa de análisis no fue posible estudiar la patología neonatal. Los datos recogidos sirvieron para describir las variables únicas relacionadas con la madre durante el embarazo y el parto, y el peso y Apgar de los recién nacidos (5).

Las modificaciones introducidas al programa facilitaron el estudio de múltiples variables para efectuar un diagnóstico de la morbimortalidad perinatal.

El estudio de la patología materna y feto-neonatal es prioritario en todo servicio obstétrico, porque su desconocimiento impide la supervisión y la aplicación de técnicas que conduzcan a la solución de los problemas.de la madre y su hijo.

En el Departamento de Obstetricia y Ginecología se han realizado tres estudios sobre letalidad perinatal (4-6-7). Los dos primeros se efectuaron gracias al funcionamiento del Comité de Mortalidad Perinatal, el cual fue fundado el 10. de febrero de 1957 y estuvo inte- grado por profesores de los Departamentos de Pediatría, Medicina Preventiva, Anatomía Patológica y Obstetricia. Diferentes factores que no son del caso mencionar aquí, impidieron que siguiera funcionando.

El último estudio de cohorte se realizó en $1976^{7}$ y se refiere a las madres que se hospitalizaron para la atención del parto. La información se recogió en el postparto inmediato.

En el presente trabajo se incluyen los datos maternos y fetoneonatales de 3.404 partos consecutivos atendidos en la Fundación Hospitalaria San Vicente de Paúl durante el año de 1982.

\section{OBJETIVOS}

\section{1.- General}

Determinar las causas de morbi-mortalidad perinatal presentadas en el Departamento de Obstetricia y Ginecología de la Facultad de Medicina de la Universidad de Antioquia (Fundación Hospitalaria San Vicente de Paúl), durante el año de 1982.

\section{2.- Específicos}

2.1 Determinar la frecuencia de cada una de las patologías encontradas en los neonatos cuyas madres hubieran presentado o no patología durante el embarazo. 
2.2 Analizar la tasa de mortalidad neonatal precoz por peso al nacer.

2.3 Analizar la mortalidad fetal y neonatal precoz en los embarazos únicos.

2.4 Analizar la tasa de mortalidad perinatal según el peso al nacer, la edad gestaciona! al parto y la consulta prenatal.

\section{METODO.}

Para la presente investigación se siguió la misma metodología del estudio sobre diagnóstico de situación perinatal, realizado en cuatro centros asistenciales de la ciudad de Medellín ${ }^{5}$.

El formulario para recoger la información fue la historia clínica perinatal presentada en el XIV Congreso Colombiano de Obstetricia y Ginecología.

Los datos registrados en la hoja del resumen estadístico perinatal fueron controlados sistemática y personalmente por uno de los autores. La información de 3.404 partos fue procesada en un computador IBM 4341, de acuerdo con los programas diseñados y previamente probados para aplicaciones de tipo biomédico.

La población atendida ingresó al Departamento de Obstetricia y Ginecología durante el año de 1982 para la culminación de su proceso reproductivo. Se incluyeron todos los embarazos únicos y gemelares, sin tener en cuenta la vía de terminación del parto, pero para el presente informe, sólo se analizaron los embarazos únicos. Se excluyeron los abortos y los embarazos ectópicos.

El tiempo promedio que permanecen las madres en el hospital es de 48 horas. Cuando los niños pesaron más de 2.500 gramos se consideró como muerte neonatal la ocurrida durante los dos primeros días; cuando los recién nacidos pesaron menos o presentaron alguna patología fueron hospitalizados en neonatología $y$, en estos casos, consideramos el período neonatal hasta el 70 . día.

Se incluyeron todas las variables mencionadas en el diagnóstico de situación perinatal, y además, para efectos del presente informe, se tuvieron en cuenta las siguientes:

\section{Edad materna}

\section{Estado civil}

Antecedentes obstétricos: Gestaciones previas -Partos anteriores -Intervalo con el último embarazo-Abortos-mortinatos y muertes neonatales.

Consulta prenatal: Edad del embarazo en la primera consulta-número de consultas-hábito de fumar y número de cigarrillos durante el presente embarazo.

Patología materna: Infección y amenaza del parto prematuro-Hemorragia: época y causa de la misma-Hipertensión crónica o inducida por el embarazo.

Otra patología: Cardiopatía-diabetes enfermedad del colágeno-neoplasias-enfermedades vasculares.

Parto: Número de partos y presentación, Edad gestacional al momento del parto,-Comienzo del parto y uso de oxitocina,-Anestesia durante el parto,-Terminación del parto,-Duración del primer período desde 4 a $5 \mathrm{cms}$.,-Estado de las membranas al ingreso $y$ tiempo de la ruptura.

Recién nacido: Sexo y estado a nacerPeso, Indice de Apgar al primero $y$ al quinto minuto-Estado del niño a la salida -Edad gestacional por el examen físico. 
Patología Neonatal: Hiperbilirrubinemia-Síndrome de dificultad respiratoria idiopática-Síndrome aspirativo-Otro síndrome de dificultad respiratoria-Patología neurológica -Anomalías congénitasInfección: tiempo de aparición y caracter ísticas. Otras: metabólicas.

Morbilidad materna: Estado a la salida-Causa de la muerte.

Definición de términos: Para el presente estudio se aceptaron los términos recomendados por la OMS Organización Mundial de la Salud, con modificaciones propuestas por la FIGO Federación Internacional de Ginecología y Obstetricia.

Se incluyeron todos los fetos y recién nacidos con un peso de 500 gramos o más, independientemente del estado al nacer.

Aborto: El feto o embrión con un peso inferior a 500 gramos y una amenorrea aproximada de 20 a 22 semanas.

Bajo peso al nacer: Se incluyeron los neonatos con un peso de 2499 gramos o menos.

Muy bajo peso al nacer: Los neonatos con peso comprendido entre 500 y 1.499 gramos.

Período Perinatal: Tiempo transcurrido entre las 28 semanas completas de embarazo hasta el 7o. día de vida extrauterina.

Recién nacido de término: Se incluyeron los neonatos productos de una gestación comprendida entre 37 y 42 semanas de amenorrea (259-293 días completos).

Prematuro o pretérmino: Se incluyeron los recién nacidos con una gestación menor de 37 semanas completas de amenorrea. (Menos de 259 días completos).
Post-término: Todos los recién nacidos con más de 42 semanas completas de amenorrea(294 días o más).

Recién nacido deprimido: Los neonatos con un índice de Apgar igual o menor de 6.

Para el presente estudio no se hizo ninguna corrección de las muertes fetales, perinatales, neonatales o maternas $y$ se incluyen todas sin tener en cuenta el lugar donde se inició el proceso morboso que desencadenó el deceso.

El número de nacidos vivos fue de $3.323(97.6 \%)$ de los cuales murieron 21 en el período neonatal. El total de muertes perinatales fue de 102 repartidas en la siguiente forma: cinco durante el embarazo, 67 en el momento del parto, 21 neonatales, y en las nueve restantes no se conoció el momento de la muerte.

El número de muertes maternas como complicación del embarazo, parto 0 puerperio fue de nueve, de las cuales tres se originaron en una hipertensión inducida por el embarazo (H.I.E.).

En el análisis de los resultados se presentan $\mathbf{2 5}$ gráficas donde se incluyen datos del embarazo, la atención prenatal, la patología materna y el parto. Del recién nacido se analiza la patología y la mortalidad.

Al final del presente informe se anexan 35 tablas que se utilizaron para la elaboración de las gráficas y que permiten al lector sacar conclusiones personales, criticar los resultados y aceptar o rechazar los mismos.

\section{RESULTADOS}

Características de la Muestra: De acuerdo con los resultados observados el grupo de gestantes estaba constituído por mujeres relativamente jóvenes, de condición socio-económica baja, de es- 
A continuación se presentan las referencias utilizadas para calcular las tasas de morbilidad y mortalidad.

REFERENCIAS DE LAS FIGURAS $19,20,21,24,25$ y TASAS

1.- Frecuencia Relativa $\%$ en la población

2.- Frecuencia Relativa $\%$ dentro de las Patologías

3.- Mortalidad Especifica por Patología

4.- Tasa \%o de Mortalidad Fetal Tardía

5.- Tasa \%o de Mortalidad Neonatal Precoz $500 \mathrm{~g}$

6.- Tasa \%o de Mortalidad Perinatal Niodificada

7.- Contribución \% a la Mortalidad

8.- Contribución $\%$ a la Mortalidad Neonatal Precoz $500 \mathrm{~g}$

$$
\begin{aligned}
& =\frac{\text { No. de Casos de la Patología } \times 100}{\text { Total de Embarazos }} \\
& =\frac{\text { No. de Casos de la Patología }}{\text { Total de las patologías }} 100
\end{aligned}
$$

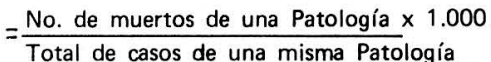

- No. de Muertes Fetales Tardías x 1.000

No. Total de Nacidos vivos más muertes Fetales Tardías

$=\frac{\text { No. de Muertes Neonatales Precoces } \supseteq 500 \mathrm{~g} \times 1.000}{\text { No. Total de Nacidos Vivos } \triangleq 500}$

$=\frac{\text { No. de Muertes Fetales Tardías más Neonatales precoces } \geq 500 \mathrm{~g}}{\text { No. Total de Nacidos Vivos } \geq 500 \mathrm{~g} \text { más Muertes Fetales Tardías }}$

$=\frac{\text { No. de Muertes en una Patología }}{\text { Total de Muertes }}$

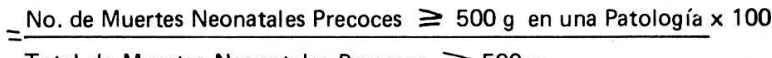

Figura I

EDAD GESTACIONAL AL PARTO

F.H.S.V. de Paủl .- Medellín 1.982

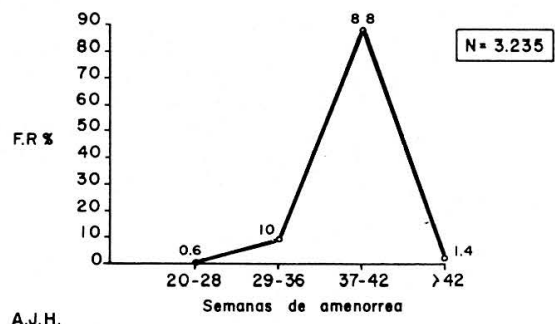

Salta a la vista que una alta proporción de las madres ingresaron con una edad gestacional comprendida entre 37 y 42 semanas, es decir, un embarazo de término.

El promedio de la edad gestacional fue de 38.7 semanas similar al encontrado en el estudio de 1981 el cual fue de 38 semanas.

El porcentaje de prematuridad $10.6 \%$, es ligeramente inferior al encontrado en el estudio anterior $(14.3 \%)$. 
casa educación, con alta proporción de primigestantes, un registro tardío de consulta prenatal, y un reducido número de controles.
La información correspondiente a las características de la muestra se presenta en los gráficos 4 y 5 y en las tablas 1, 3, 7,11 y 12

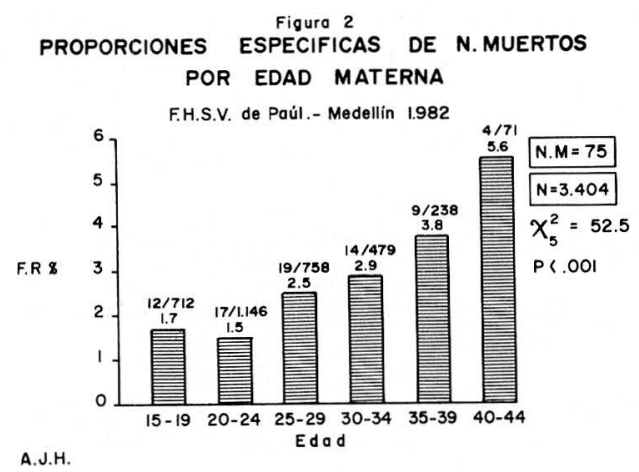

A.J.H.

Figura 3

PROPORCIONES ESPECIFICAS DE N. MUERTOS
POR PARIDAD

F.H.S.V. de Paúl.- Medellin 1.982

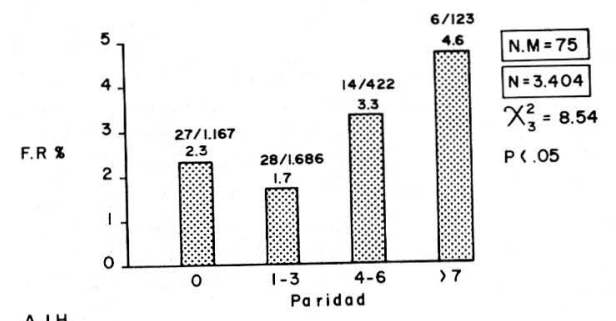

El porcentaje de nacidos muertos aumen ta consistentemente a partir de los 20 años, desde el $1.5 \%$ hasta el $5.6 \%$ en el grupo de 40 a 44 años.

En el grupo de madres más jovenes (15 a 19 años) el porcentaje de mortalidad fue ligeramente superior $(1.7 \%)$ al observado en el de 20 a $24(1.5 \%)$.
La mortalidad aumenta progresivamente con la paridad a partir del grupo de 1 a 3 partos, en el que la mortalidad es de $1.7 \%$, hasta el $4.7 \%$ en el grupo con paridad mayor o igual a 7 . La mortalidad en las nulíparas es mayor $(2.3 \%)$ que la encontrada en el grupo de 1 a 3.


Figura 4

\section{$N^{*}$ DE CONTROLES PRENATALES}

F.H.S.V. de Paúl.- Medellin 1.982

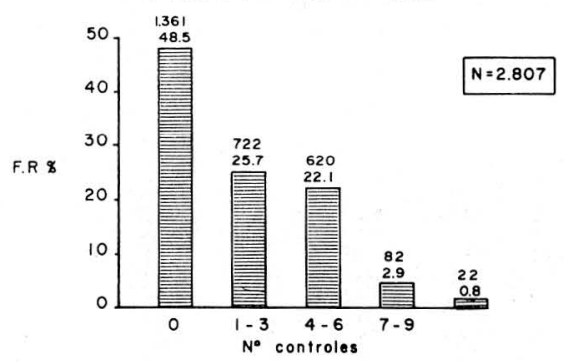

Figura 5

EDAD GESTACIONAL EN LA PRIMERA CONSULTA

F.H.S.V.de Poúl.-Medellin 1.982

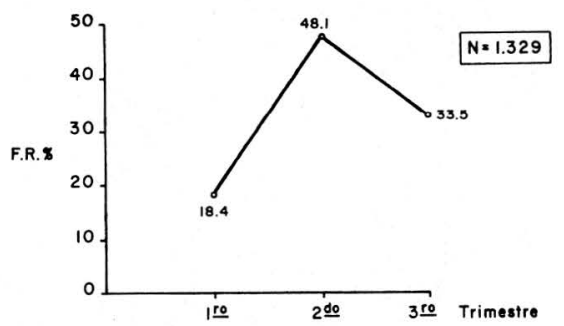

A.J.H.

\begin{abstract}
Prácticamente la mitad de las embarazadas no asistió a la consulta prenatal, $(48.5 \%)$ lo que concuerda con lo encontrado en $1981(47 \%)$. De aquéllas que tuvieron control prenatal, cerca del $50 \%$ consultaron entre una y tres veces.

De las gestantes que asistieron a consulta prenatal un poco menos de la quinta parte $(18.4 \%)$ la iniciaron en el primer trimestre, cerca de la mitad $(48.1 \%)$, en el segun do, y la tercera parte $(33.5 \%)$ en el último.
\end{abstract}

PATOLOGIA MATERNA

Amenaza de parto prematuro

En el grupo de madres estudiadas 138 $(5.4 \%)$ presentaron amenaza de parto prematuro. Esta patología fue más frecuente en aquéllas con paridad cero que en el resto de las pacientes.
Figura 6

PROPORCIONES ESPECIFICAS DE A.P. PREMATURO POR PARIDAD

F.H.S.V. de Paúl.-Medellín 1.982

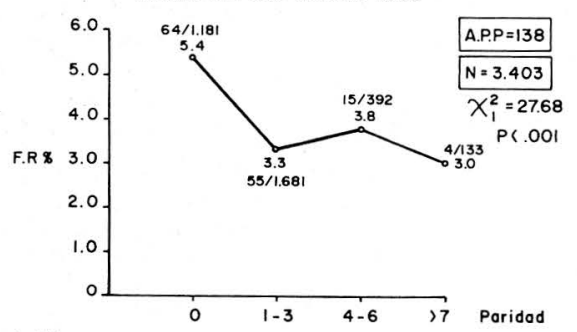

A.J.H. 
Figura 7

DISTRIBUCION PORCENTUAL POR EDAD DE LAS PACIENTES CON H.I.E.

F.H.S.V. de Paúl.- Medellin 1.982

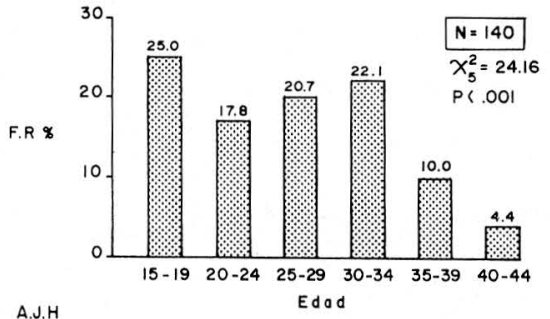

A.J.H

Figura 8

DISTRIBUCION PORCENTUAL DE LA PARIDAD

EN LAS PACIENTES CON H.I.E.

F.H.S.V. de Paúl.- Medellín 1.982

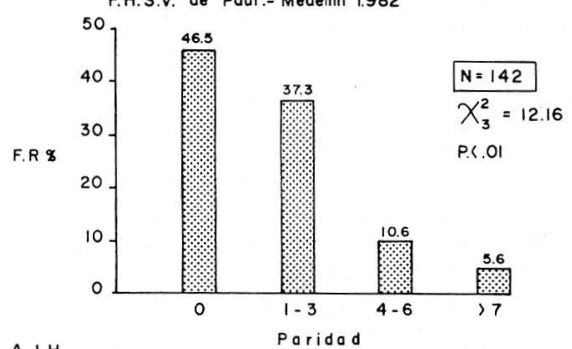

A.J.H.
Figura 80

PROPORCIONES ESPECIFICAS DE PACIENTES CON

H.I.E POR PARIDAD

F.H.S.V. de Paúl.-Medellin 1.982

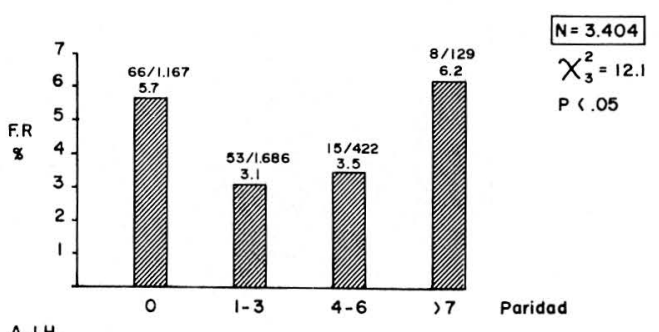

A.J.H.

Figura $8 \mathrm{~b}$.

PROPORCIONES ESPECIFICAS DE H.I.E. POR EDAD

MATERNA

F.H.S.V. de Paúl.- Medellin 1.982

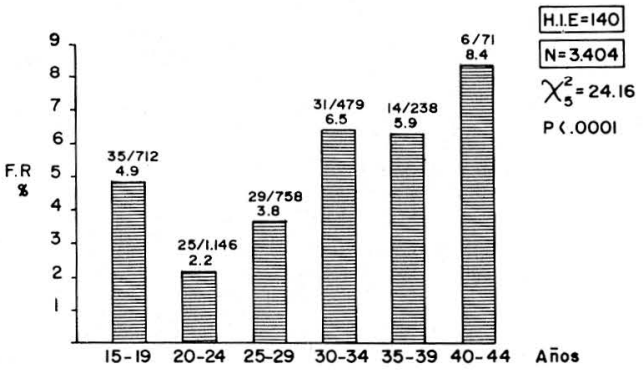

A.J.H.

Hipertensión Inducida por el Embarazo (H.I.E.)

Al estudiar el comportamiento de la HIE de acuerdo con la edad y la paridad (figuras $7,8,8 \mathrm{~A}$ y $8 \mathrm{~B}$ ), se observa en la distribución porcentual de la edad materna que el $25.0 \%$ corresponde al grupo de $15 \cdot 19$ años y el $4.4 \%$ al de mayor edad 40 - 44 años. Sin embargo, al analizar el com portamiento de la misma variable de acuerdo con las proporciones específicas (riesgo), se observa que la incidencia más alta se presenta en el grupo de 40.44 años $(8.4 \%)$ y la menor $(2.2 \%)$ en el de 20 - 24 años como puede apreciarse en la figura $8 \mathrm{~B}$.

Por otra parte en el comportamiento de acuerdo con la paridad se encontró una incidencia igual en las multíparas y las primíparas $6.2 \%$ y $5.7 \%$ respectivamente y una incidencia menor en los grupos de 1.3 y 4.6 partos $(3.1$ y $3.5 \%)$ respectivamente (figura $8 \mathrm{~A})$. 


\section{RECIEN NACIDOS}

En el gráfico se aprecia la estrecha correlación que existe entre el bajo peso al nacer y el número de cigarrillos fumados diariamente durante la gestación. El porcentaje de niños con bajo peso entre las madres que no fumaron durante la gestación fue de $11.3 \%$, fracción que aumenta progresivamente hasta el $26.9 \%$ en aquéllas que fumaron 16 ó más cigarrillos por día. En dicho resultado se advierte un aumento notorio cuando se pasa de la categoría de 6 a 10 cigarrillos a la de 11 y más.

Al categorizar el hábito de fumar en 0,1 . 10 y 11 y más cigarrillos, se obtuvieron diferencias significativas en la proporción de niños con bajo peso $\left(X_{2}^{2}=21.7, p \quad 001\right)$.

En el gráfico No. 10 se presenta la distribución del peso de los recién nacidos. De acuerdo con los datos obtenidos el 13\% de los neonatos presentaron un bajo peso (menos de $\mathbf{2 5 0 0}$ gramos), el $\mathbf{8 4 . 3} \%$ tuvie ron un peso adecuado, y el $2.7 \%$ presentaron caracter ísticas macrosómicas.

Al estudiar el estado del recién nacido de acuerdo con el índice de Apgar se observa una depresión (Apgar $\leq 6$ ) que fue progresivamerite más frecuente en la medida que se utilizaron procedimientos más com. plicados para la atención del parto.

\section{Figura 9}

\section{PROPORCIONES ESPECIFICAS DE BAJO PESO}

AL NACER POR HABITO DE FUMAR

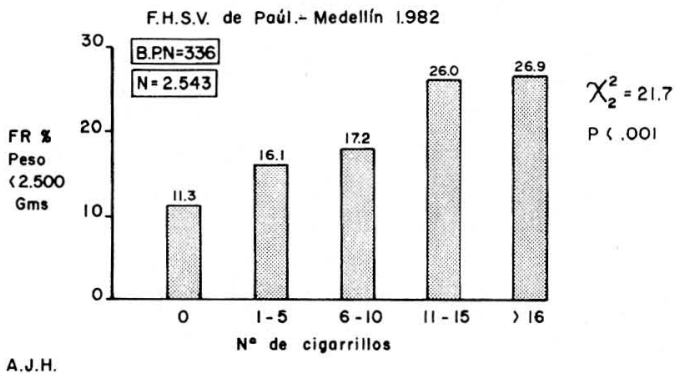

A.J.H.

\section{DISTRIBUCION PORCENTUAL DEL PESO DE LOS RECIEN NACIDOS}

Figuro 10

F.H.S.V. de Poúl.- Medellin 1.982

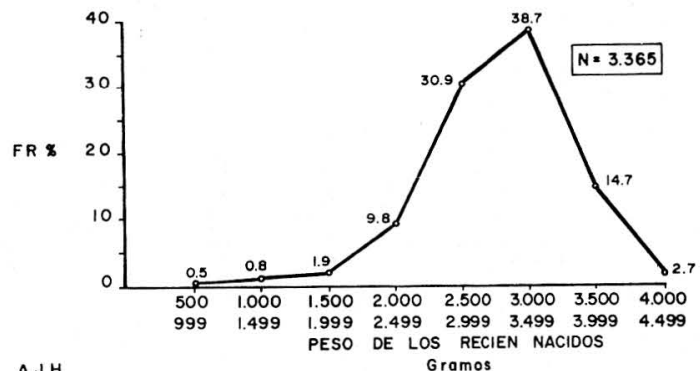

Figura II

PROPORCIONES ESPECIFICAS DE NEONATOS CON APGAR BAJO A LOS 5 MIN. POR TERMINACION DEL PARTO

F.H.S.V. de Paúl.-Medellin 1.982

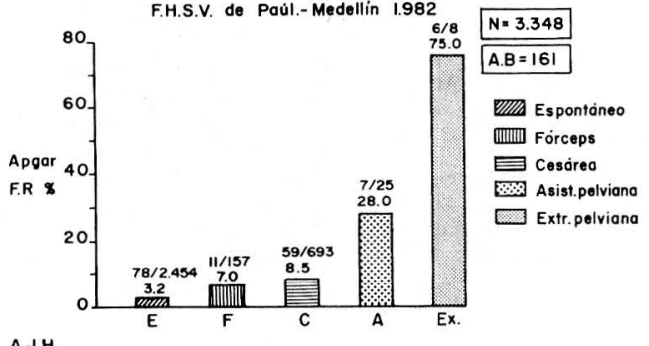

A.J.H. 


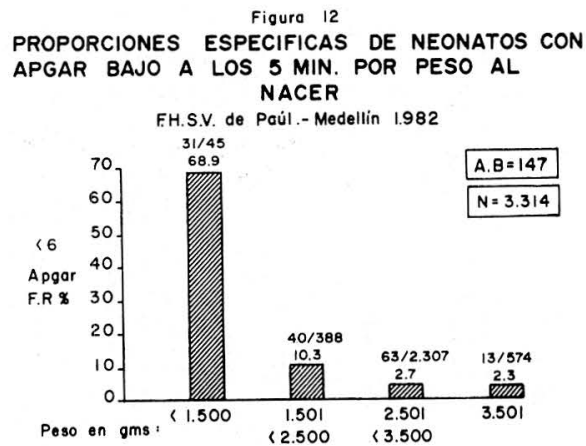

A.J.H.

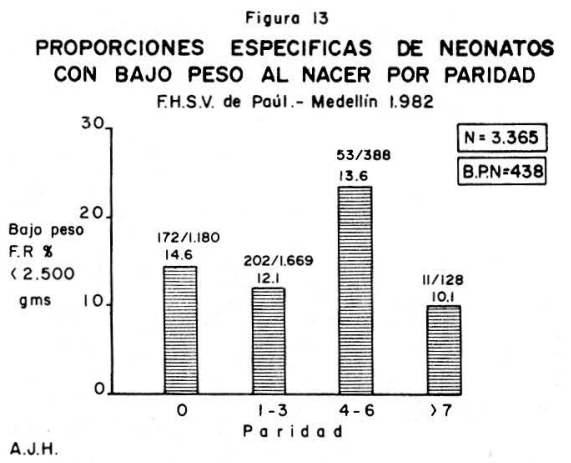

Figura 14

PROPORCIONES ESPECIFICAS DE NEONATOS

CON BAJO PESO POR TIPO DE PARTO

F.H.S.V. de Paúl. - Medellin 1.982

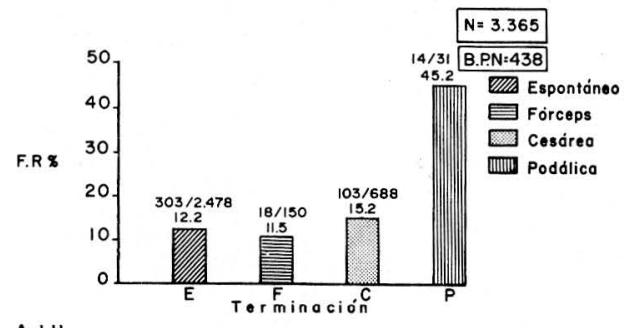

A.J.H.

\begin{abstract}
La clasificación de este mismo indice de acuerdo con el peso al nacer mostró un alto porcentaje de neonatos deprimidos $(68.9 \%)$ en aquéllos que pesaron menos de $1500 \mathrm{gra}$. mos, cifra que fue significativamente menor para los niños con un peso superior.
\end{abstract}

El análisis del bajo peso al nacer de acuerdo con la paridad señala un mayor riesgo para las gestantes con antecedentes de 4 a 6 partos y un menor riesgo para aquéllas con una paridad mayor de 7 . No obstante, la relación entre las 2 variables no es consistente.

El bajo peso al nacer (B.P.N.) se observó mucho más frecuentemente en los niños que nacieron en presentación podálica que en aquéllos atendidos con otros procedimientos. En éstos, los neonatos de bajo peso extraídos por cesárea $(15.9 \%)$ fueron un poco más frecuentes que los de parto espontáneo $(12.2 \%)$ y fórceps $(11.5 \%)$ respectivamente. 
La relación observada entre el número de nacidos muertos y la paridad señala al grupo de 1 a 3 partos como el de menor ries. go, y al de 7 ó más como el de mayor riesgo. El riesgo aumenta progresivamente a partir del grupo de paridad 1-3; el grupo de primigestantes presenta una proporción al. go mayor $(2.3 \%)$ de nacidos muertos que el de paridad $1.3(1.7 \%)$.

La mortalidad fetal (intermedia y tardia) de acuerdo con el número de controles prenatales fue mayor en el grupo con 10 y más controles, grupo al cual sigue en su orden el de aquéllas que no asistieron a ninguna consulta y cuya mortalidad fue la mitad de la encontrada en el grupo anterior, En el grupo de las que asistieron entre 1 y 9 consultas el porcentaje de mortalidad fetal fue a su vez la mitad de aquéllas que no. asistieron a ninguna consulta.

En el grupo de 10 y más consultes prenatales están incluidas aquéllas embarazadas con un riesgo muy elevado y que generalmente acuden al programa de alto riesgo obstétrico del Departamento de Obstetricia y Ginecología.

Faundes et al (1) encontró un buen resultado perinatal cuando la consulta prenatal se inició antes del quinto mes, el número de controles fue de cinco y el médico sólo realizó el primero.
Figura 15

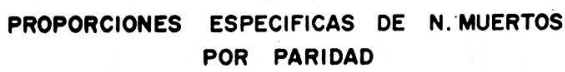

F.H.S.V. de Paúl.-Medellin 1.982

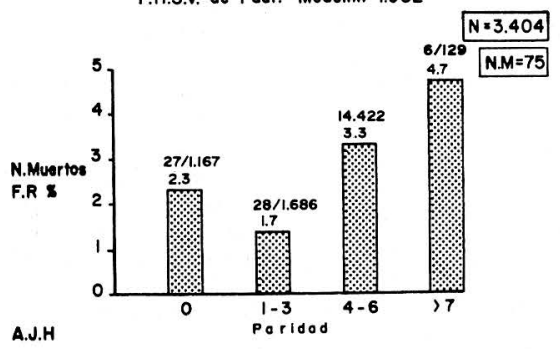

A.J.H

Figura 16

TASA DE MORTALIDAD FETAL SEGUN C.PRENATALES

F.H.S.V. de Paúl.-Medellin 1.982

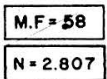

F.R \&

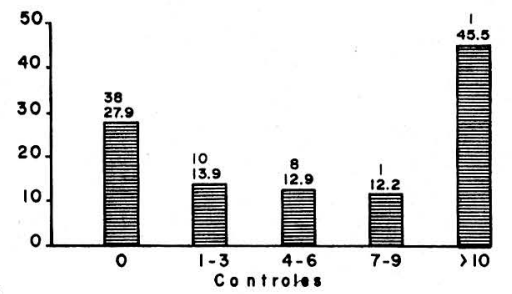

A.J.H.

Figura 17

\section{PROPORCIONES ESPECIFICAS DE N. MUERTOS \\ POR PESO}

F.H.S.V. de Paúl.- Medellín 1.982

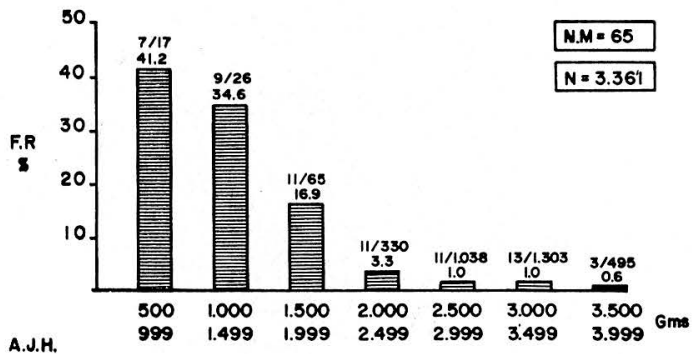

A.J.H.
Al observar el porcentaje de mortinatos segun el peso al nacer se advierte que en los neonatos con muy bajo peso (500. 999 gramos), se mueren 2 de cada 5 recién nacidos vivos $(41.2 \%)$. Esta frecuencia disminuye progresivamente y para los de $\mathbf{2 5 0 0}$ gramos en adelante, el porcentaje de muertos es del $1 \% 0$ menos. 


\section{Figura 18 \\ PROPORCIONES ESPECIFICAS DE N.MUERTOS \\ POR TIPO DE PARTO}

F.H.S.V. de Paúl.- Medellin 1.982

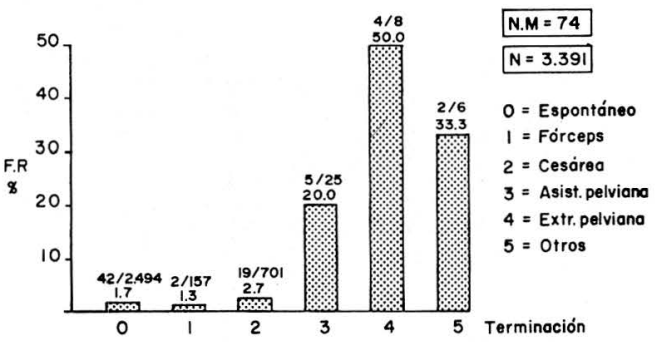

Figura 19

FRECUENCIA DE LAS PATOLOGIAS NEONATALES

F.H.S.V. de Paúl.- Medellín 1.982

$P=831 \quad N=3.401$

INFECCION

HIPERBILIRRUBINEMIA

OTRA PATOLOGIA

S. ASPIRATIVO

PAT. NEUROLOGICA

S.D.R.I.

ANOMALIA CONGENITA

OTRO S.D.R.

A.J.H.

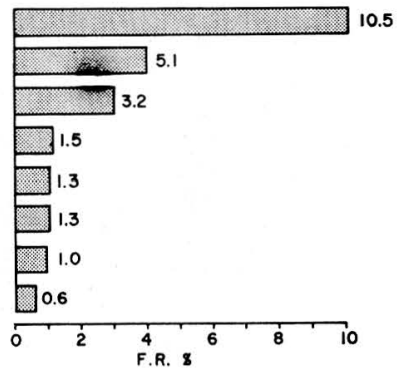

Figuro 20

DISTRIBUCION PORCENTUAL DE LA MORBILIDAD

F.H.S.V. de Paúl.- Medellín 1.982

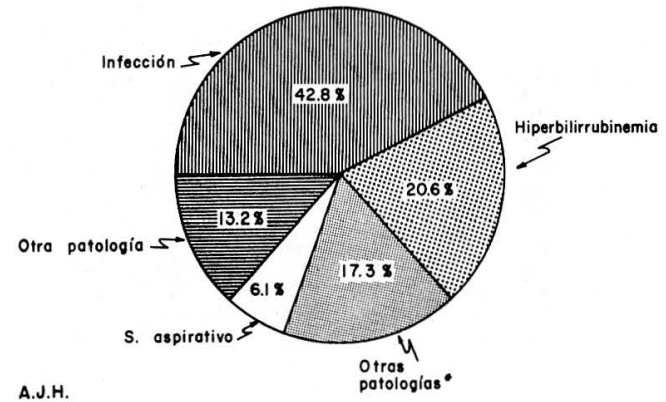

La frecuencia de mortinatos guarda una estrecha relación con la complejidad de los procedimientos utilizados para la atención del parto tal como se observa en el gráfico 18: el número de nacidos muertos es mayor cuando se realiza la extracción pelviana seguida de la asistencia de la misma y cuando se efectúan combina. ciones de procedimientos.

La distribución de la incidencia de las patologias neonatales muestra que los procesos infecciosos ocupan la primera posición con el $10.5 \%$ de casos observados en el total de nacimientos. La hiperbilirrubinemia, segunda patología en importancia, se presentó en el $5.1 \%$ de todos los neonatos.

En conjunto el $24.5 \%$ de todos los neonatos presentaron alguna patología.
La distribución porcentual de la morbilidad señala que algo más de las $2 / 5$ partes de todas las patologías son de tipo infeccioso, y cerca de una quinta parte corresponde a la hiperbilirrubinemia.

- En este grupo están incluidas las siguientes patologías: patología neurológica $5.3 \%$, sindrome de dificultad respiratoria idiopático $5.3 \%$, anomalías congénita $4.1 \%$ y otro síndrome de dificultad respiratoria $2.6 \%$. 
En el estudio de la letalidad, las anomalías congénitas presentan la mayor tasa (147 por mil). Un lugar preponderante está ocupado por el síndrome de dificultad respiratoria idiopático (SDRI) y por la patología neurológica (68.2 por mil). Por otra parte, cabe señalar la baja letalidad en las infecciones ( 8.4 por mil casos), que ocupan el último lugar en este contexto.

La infección neonatal, de acuerdo con lo observado en el gráfico 22, fue más frecuente a medida que aumentó el tiempo transcurrido entre la ruptura de las mem. branas y el momento del parto: para los casos con un período de 4 o menos horas la infección se presentó en 1 de cada 10 niños en cambio en aquéllos con un período de 24 o más horas la ocurrencia de dicha patología se observó en 3 de cada 10 nacimientos.

\section{Figura 21}

TASA DE LETALIDAD POR CADA PATOLOGIA

F.H.S.V. de Paúl.- Medellin 1.982

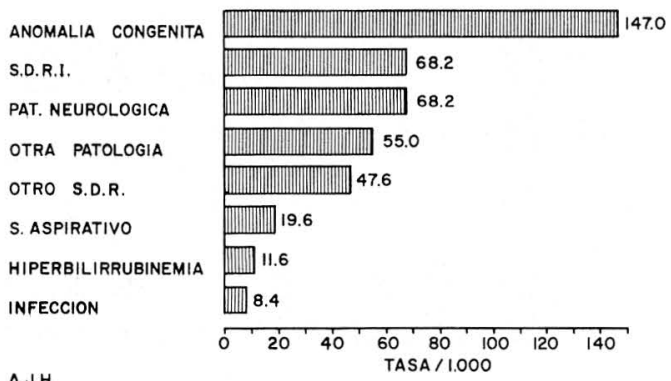

Figura 22

PROPORCIONES ESPECIFICAS DE LA INCIDENCIA DE DE INFECCION NEONATAL POR EL TIEMPO DE R.

DE MEMBRANAS

F.H.S.V. de Paúl. - Medellín 1.982

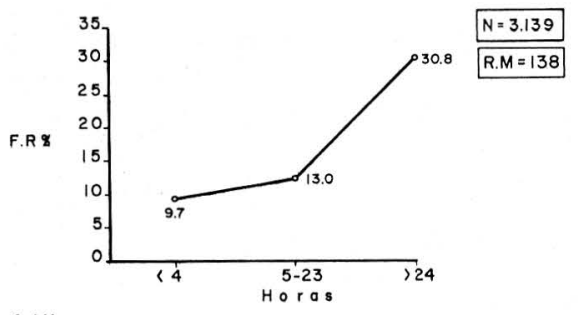

A.J.H.

Figura 23

CARACTERISTICAS DE LA INFECCION NEONATAL

F.H.S.V. de Paúl.-Medellin 1.982

Una clasificación de la infección de acuerdo con el momento de la aparición y su extensión revela que en el $72 \%$ de los casos ésta se presentó entre el segundo y el séptimo día y fue de tipo localizado. Quizás este hecho explique la baja letalidad observada en aquéllos casos que presentaron infección. 
FIGURA 24

PROPORCIONES ESPECIFICAS DE N. MUERTOS NEONATALES POR PESO AL NACER

F.H.S.V. de Paúl.- Medellín 1.982

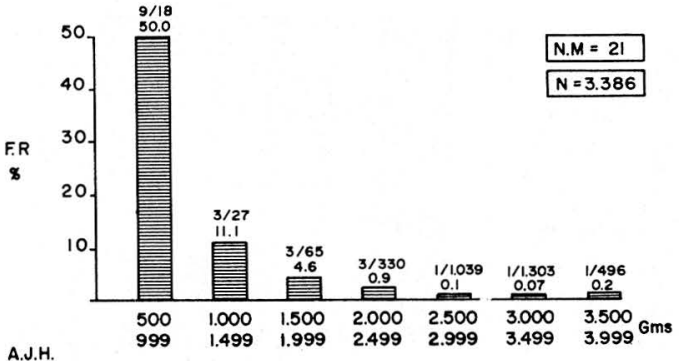

El número de muertes neonatales está en relación directa con el peso al nacer. $\mathrm{Pa}$ ra los niños de muy bajo peso (500-999 gramos) el porcentaje de mortalidad neonatal fue de $50 \%$. En la siguiente categoria (1000-1499 gramos) este porcentaje es del $11.1 \%$. En la categoría de 1500 -1999 gramos el porcentaje es del $4.6 \%$ y en las restantes (2000 gramos y más) el porcentaje es inferior al $1 \%$.

Figura 25

MORTALIDAD NEONATAL PRECOZ SEgUN PESO AL NACER

F.H.S.V. de Paúl.- Medellin 1.982

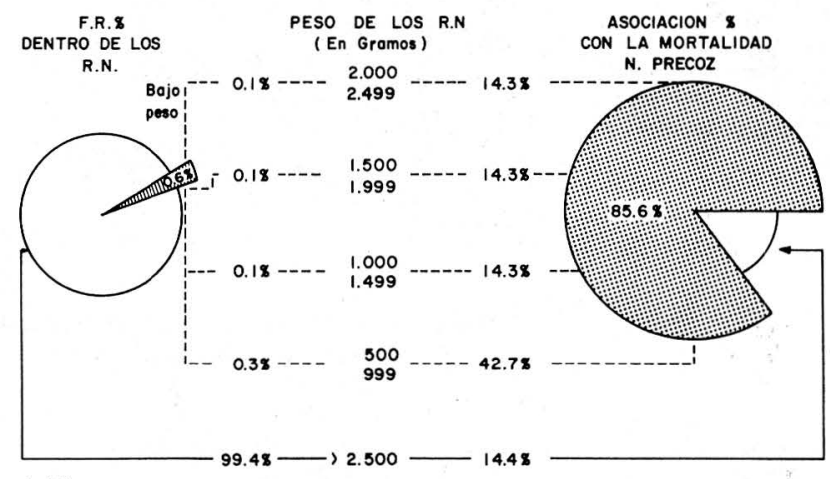

A.J.H. 


\section{DISCUSION}

La historia clínica perinatal que se utilizó para recoger la información facilitó la sistematización de los datos maternos y feto neonatales más importantes. El modelo presentado ha sido probado previamente en cuatro centros asistenciales de la ciudad de Medellín 5 $y$ en otras maternidades de América Latina $^{2}$.

El diligenciamiento completo es una responsabilidad del personal médico $y$ paramédico, como parte de la atención de la mujer durante el proceso reproductivo.

En el presente estudio se incluyeron todos los fetos y recién nacidos con un peso superior a 500 gramos y no se depuró la mortalidad perinatal.

El porcentaje de prematuridad (10.6\%) es inferior en un $50 \%$ al encontrado en 1957 el cual fue de $20.0 \%$.

El porcentaje de madres que asistieron a control prenatal fue de $48.5 \%$ y solo el $18.4 \%$ de las que tuvieron alguna consulta, iniciaron dicho control en el primer trimestre. Estamos muy lejos de alcanzar las metas ideales propuestas por la OMS cuando considera que un buen control prenatal es aquel que se inicia antes del tercer mes, que el total de consultas debe fluctuar entre 8 y 12 y que el médico participa siempre en la primera consulta y en varios controles ${ }^{3}$. Se necesitaron 25 años para incrementar el porcentaje de consulta prenatal, si comparamos las cifras respectivas de $16.1 \%$ en 1957 y la que se encontró en el presente estudio $(51.5 \%)$.

El porcentaje de $13 \%$ del bajo peso al nacer (menos de 2.500 gramos), es superior al valor medio $(9 \%)$ informado en la investigación prospectiva coordinada por el CLAP en 59 maternidades públicas de 11 países de América Latina sobre un total de 333.794 partos consecutivos ${ }^{1}$.
La tasa global de mortalidad perinatal (a partir de los 500 gramos) fue de 27.4 por mil; esta cifra es $4 \frac{1}{2}$ veces menor que la encontrada en la misma Institución durante el año de $1957(119 \%)$ $y$ ligeramente inferior a los hallazgos de Latinoamérica ${ }^{1}$.

La asociación del bajo peso al nacer con las muertes neonatales es de $86.5 \%$.

\section{CONCLUSIONES}

1. La tasa de mortalidad fetal tardía es de $21.3 \%$.

2. La tasa de mortalidad neonatal precoz es de $6.3 \%$.

3. La tasa global de mortalidad perinatal es de $27.4 \%$.

4. La tasa de mortalidad fetal (intermedia y tardía) en las madres que no asistieron a consulta prenatal es de $(27.9 \%$ o, .

5. El porcentaje de las embarazadas que no asistieron a consulta prenatal continúa elevado (48.5\%).

6. El primer control prenatal aún se realiza en etapas tardías, $48.1 \%$ en el segundo trimestre y $33.5 \%$ en el último.

7. La hipertensión inducida por el embarazo se encuentra en el $4.2 \%$ de todos los casos en el momento del ingreso.

8. La hipertensión inducida por el embarazo es igualmente frecuente en las multíparas $(6.2 \%)$ que en las primiparas $(5.7 \%)$.

9. El índice de prematuridad es de $10.6 \%$.

10. El índice del bajo peso al nacer es de $13.0 \%$.

11. La incidencia de cesárea es de $20.7 \%$

12. La infección fue la primera causa de patología neonatal $(10.5 \%)$. 
13. Las anomalías congénitas producen la mayor letalidad neonatal $(147 \%$ ) y la infección menor $(8.4 \%$ ) .

14. El porcentaje de mortinatos es significativamente mayor $(96.0 \%)$ en los recién nacidos de bajo peso.

15. El porcentaje de muertes neonatales en los recién nacidos con bajo peso es de $85.6 \%$.

16. La tasa de mortalidad materna es de 27.0 por 10.000 nacidos vivos.

\section{RECOMENDACIONES}

Una tarea prioritaria entre nosotros debe ser el estudio de las causas que influyen para que las embarazadas que acuden en demanda de servicios a la Fundación Hospitalaria San Vicente de Paúl, no asistan con mayor frecuencia a la consulta prenatal.

El control prenatal debe ser precoz, frecuente $y$ de óptima calidad para que pueda prevenir la amenaza de parto prematuro, la HIE y el bajo peso al nacer.

La infección neonatal como causa principal de la patología neonatal debe despertar el interés de pediatras y obstetras para buscar las medidas correctivas.

El análisis crítico y permanente de todas las muertes fetales $y$ neonatales debe ser el objetivo principal de un comité de mortalidad perinatal, el cual debe integrarse con personal de los Departamentos de Obstetricia y Ginecología, Pediatría, Anatomía Patológica y Medicina Preventiva.

\section{RESUMEN}

Se analizan los datos maternos y fetoneonatales de 3.404 partos consecutivos ocurridos en el Departamento de Obstetricia y Ginecología de la Facultad de Medicina de la Universidad de Antioquia (Fundación Hospitalaria San Vicente de Paúl), durante el año de 1982. Se hace énfasis en la morbi-mortalidad perinatal.

En el presente estudio sólo se incluyen los recién nacidos productos de embarazos únicos. El total de nacidos vivos fue de $3.323(97.6 \%)$. El $82.7 \%$ de las muertes fetales ocurrieron durante el parto. La principal causa de morbilidad neonatal fue la infección $(10.5 \%$ ) y la primera causa de letalidad neonatal la ocıpó la anomalía congénita (147 x 1.000).

Se incluyeron todos los recién nacidos con un peso superior a 500 gramos y se analizan los datos relacionados con el embarazo, parto y período neonatal.

Durante el mismo lapso murieron nueve madres de causa obstétrica directa $(0.3 \%)$.

\section{PERINATAL MORBI-MORTALITY}

\section{SUMMARY}

This article analyzes maternal, fetal and neo-natal data related to 3.404 consecutive deliveries in San Vicente de Paul Hospital, Ob-Gyn Department, Antioquia University, during 1982, with special emphasis in perinatal morbidity and mortality.

This study includes offspring of single pregnancies only. The total number of live births was $3,323(97.6 \%) .82 .7 \%$ of fetal losses took place during delivery.
I he main cause of neo-natal morbidity was infection $(10.5 \%)$ and the first cause of mortality was congenital abnormality $(147 \times 1.000)$.

The study takes into account all the newborn weighing more than 500 grams, and analyzes data related to pregnancy, delivery and the neo-natal phase.

During the same period, nine mothers died of direct obstetrical complications $(0.3 \%)$. 


\section{ANEXO}

TABLA 1

DISTRIBUCION DE LA EDAD DE LAS EMBARAZADAS

\begin{tabular}{|c|r|c|}
\hline Edad & No. & \multicolumn{1}{c|}{$\%$} \\
\hline $15-19$ & 683 & 20.2 \\
$20-24$ & 1.146 & 33.9 \\
$25-29$ & 762 & 22.5 \\
$30-34$ & 480 & 14.2 \\
$35-39$ & 239 & 7.1 \\
$40-44$ & 71 & 2.1 \\
\hline Total & 3.381 & 100.0 \\
\hline
\end{tabular}

TABLA 2

ESTADO CIVIL

\begin{tabular}{|l|c|c|}
\hline & No. & $\%$ \\
\hline Casadas & 1.708 & 50.2 \\
Unión Libre & & \\
estable & 1.198 & 35.2 \\
Solteras & 495 & 14.6 \\
\hline Total & 3.401 & 100.00 \\
\hline
\end{tabular}

TABLA 3

NUMERO DE PARTOS PREVIOS

(VAGINALES Y CESAREAS) AL

PRESENTE EMBARAZO

\begin{tabular}{|c|r|r|}
\hline & No. & $\%$ \\
\hline 0 & 1.098 & 32.3 \\
$1 \cdot 3$ & 1.542 & 45.3 \\
$4-6$ & 534 & 15.7 \\
$7 \cdot 9$ & 228 & 6.7 \\
\hline Total & 3.402 & 100.0 \\
\hline
\end{tabular}

TABLA 4

INTERVALO INTERGENESICO (ENTRE

EL FINAL DEL ULTIMO EMBARAZO,

PARTO O ABORTO Y EL PRIMER DIA DE LA MENSTRUACION) CORRESPONDIENTE AL EMBARAZO ACTUAL

\begin{tabular}{|c|r|r|}
\hline Años & No. & $\%$ \\
\hline 0 & 1.167 & 56.5 \\
$1-2$ & 499 & 24.2 \\
$3-5$ & 258 & 12.5 \\
6 & 141 & 6.8 \\
\hline Total & 2.065 & 100.0 \\
\hline
\end{tabular}

TABLA 5

TERMINACION DEL PARTO SEGUN LA EDAD MATERNA

\begin{tabular}{|c|c|c|c|c|c|c|c|c|c|c|c|c|c|}
\hline \multirow[t]{3}{*}{ Tipo Parto } & \multicolumn{13}{|c|}{ EDAD } \\
\hline & \multicolumn{2}{|c|}{$15 \cdot 19$} & \multicolumn{2}{|c|}{$20 \cdot 24$} & \multicolumn{2}{|c|}{$25 \cdot 29$} & \multicolumn{2}{|c|}{$30 \cdot 34$} & \multicolumn{2}{|c|}{$35 \cdot 39$} & \multicolumn{2}{|c|}{$40 \cdot 44$} & \multirow[b]{2}{*}{ Total } \\
\hline & No. & $\%$ & No. & $\%$ & No. & $\%$ & No. & $\%$ & No. & $\%$ & No. & $\%$ & \\
\hline Espontáneo & 478 & 70.4 & 855 & 74.8 & 568 & 75.2 & 369 & 77.2 & 162 & 68.0 & 47 & 66.2 & 2.479 \\
\hline Fórceps & 56 & 8.2 & 48 & 4.2 & 30 & 4.0 & 14 & 2.9 & 6 & 2.5 & 0 & 0.0 & 154 \\
\hline Cesárea & 137 & 20.2 & 230 & 20.1 & 152 & 20.1 & 90 & 18.8 & 65 & 27.3 & 23 & 32.4 & 697 \\
\hline $\begin{array}{l}\text { Asistencia Ex- } \\
\text { tracción Pólvica }\end{array}$ & 7 & 1.2 & 10 & 0.9 & 5 & 0.7 & 5 & 1.0 & 5 & 2.2 & 1 & 1.4 & 33 \\
\hline TOTAL & 678 & 100.0 & 1.143 & 100.0 & 755 & 100.0 & 478 & 100.0 & 238 & 100.0 & 71 & 100.0 & 3.363 \\
\hline
\end{tabular}


TABLA 6

EDAD GESTACIONAL AL PARTO SEGUN INTERVALO INTERGENESICO

\begin{tabular}{|c|r|r|r|r|r|}
\hline \multirow{2}{*}{$\begin{array}{c}\text { Edad gestacional } \\
\text { en semanas }\end{array}$} & \multicolumn{5}{|c|}{ INTERVALO EN AÑOS } \\
\cline { 2 - 6 } & 0 & 1.3 & $4-6$ & $>6$ & Total \\
\hline $20-28$ & 13 & 3 & 1 & 0 & 17 \\
$29-36$ & 237 & 45 & 22 & 10 & 314 \\
$37-42$ & 2.066 & 416 & 220 & 120 & 2.822 \\
$>42$ & 27 & 9 & 4 & 6 & 46 \\
\hline Total & 2.343 & 473 & 247 & 136 & 3.199 \\
\hline
\end{tabular}

TABLA 7

EDAD MATERNA POR PARIDAD

\begin{tabular}{|c|c|c|c|c|c|c|c|c|}
\hline \multirow[b]{3}{*}{ Paridad } & \multicolumn{8}{|c|}{ EDAD MATERNA EN ANNOS } \\
\hline & \multicolumn{2}{|c|}{$15 \cdot 24$} & \multicolumn{2}{|c|}{$25 \cdot 34$} & \multicolumn{2}{|c|}{$35 \cdot 44$} & \multirow[b]{2}{*}{ Total } & \multirow[b]{2}{*}{$\%$} \\
\hline & No. & $\%$ & No. & $\%$ & No. & $\%$ & & \\
\hline 0 & 958 & 51.6 & 185 & 14.9 & 24 & 7.8 & 1.167 & 34.3 \\
\hline $1 \cdot 3$ & 840 & 45.2 & 755 & 61.0 & 91 & 29.4 & 1.686 & 49.5 \\
\hline $4 \cdot 6$ & 60 & 3.2 & 252 & 20.4 & 110 & 35.6 & 422 & 12.4 \\
\hline $7>$ & 0 & 0.0 & 45 & 3.7 & 84 & 27.2 & 129 & 3.8 \\
\hline Total & 1.858 & 100.0 & 1.237 & 100.0 & 309 & 100.0 & 3.404 & 100.0 \\
\hline
\end{tabular}

TABLA 8

EDAD GESTACIONAL AL PARTO

\begin{tabular}{|l|r|r|}
\hline Semanas & No. & \multicolumn{1}{|c|}{$\%$} \\
\hline $20-28$ & 19 & 0.6 \\
$29-36$ & 324 & 10.0 \\
$37-42$ & 2.844 & 87.9 \\
$>42$ & 48 & 1.5 \\
\hline Total & 3.235 & 100.0 \\
\hline
\end{tabular}


TABLA 9

EDAD GESTACIONAL AL PARTO SEGUN LA TERMINACION

\begin{tabular}{|l|r|r|r|r|r|}
\cline { 2 - 6 } \multicolumn{1}{c|}{} & \multicolumn{5}{c|}{ EDAD GESTACIONAL } \\
\cline { 2 - 7 } TERMINACION & $20 \cdot 28$ & $29 \cdot 36$ & $37-42$ & $>42$ & Total \\
\hline Espontáneo & 11 & 206 & 2.107 & 33 & 2.357 \\
Fórceps & - & 12 & 134 & 1 & 147 \\
Cesárea & 5 & 87 & 555 & 11 & 658 \\
Asistencia Pelviana & 1 & 4 & 16 & - & 21 \\
Extracción Pelviana & - & 3 & 2 & - & 5 \\
Otras & - & 2 & - & 1 & 3 \\
\hline Total & 17 & 314 & 2.814 & 46 & 3.191 \\
\hline
\end{tabular}

TABLA 10

TERMINACION DEL PARTO SEGUN LA PARIDAD

\begin{tabular}{|c|c|c|c|c|c|c|c|c|c|c|}
\hline \multirow[b]{3}{*}{ TERMINACION } & \multicolumn{10}{|c|}{ PARIDAD } \\
\hline & \multicolumn{2}{|c|}{0} & \multicolumn{2}{|c|}{$1 \cdot 3$} & \multicolumn{2}{|c|}{$4 \cdot 6$} & \multicolumn{2}{|c|}{$7>$} & \multirow[b]{2}{*}{ TOTAL } & \multirow[b]{2}{*}{$\%$} \\
\hline & No. & $\%$ & No. & $\%$ & No. & $\%$ & No. & $\%$ & & \\
\hline Espontáneo & 731 & 64.1 & 1.330 & 78.9 & 333 & 84.9 & 86 & 66.7 & 2.480 & 74.1 \\
\hline Fórceps Bajo & 70 & 6.1 & 43 & 2.6 & 5 & 1.3 & 3 & 2.3 & 121 & 3.6 \\
\hline Fórceps Medio & 11 & 1.0 & 5 & 0.2 & 0 & 0.0 & 0 & 0.0 & 16 & 0.5 \\
\hline Cesárea Electiva & 86 & 7.5 & 101 & 6.0 & 23 & 5.9 & 10 & 7.7 & 220 & 6.6 \\
\hline Cesárea Intraparto & 236 & 20.6 & 187 & 11.2 & 26 & 6.6 & 28 & 21.7 & 477 & 14.2 \\
\hline Asistencia Pelviana & 6 & 0.5 & 14 & 0.8 & 3 & 0.9 & 2 & 1.6 & 25 & 0.8 \\
\hline Extracción Pelviana & 0 & 0.0 & 5 & 0.2 & 1 & 0.2 & 0 & 0.0 & 6 & 0.1 \\
\hline Otras & 4 & 0.2 & 1 & 0.1 & 1 & 0.2 & 0 & 0.0 & 6 & 0.1 \\
\hline Total & 1.144 & 100.0 & 1.686 & 100.0 & 392 & 100.0 & 129 & 100.0 & 3.351 & 100.0 \\
\hline
\end{tabular}

TABLA 11

CONTROL PRENATAL SEGUN LOS TRIMESTRES

\begin{tabular}{|l|r|r|}
\hline TRIMESTRE & No. & \multicolumn{1}{|c|}{$\%$} \\
\hline Sin Control & 1.362 & 50.6 \\
Primero & 245 & 9.1 \\
Segundo & 640 & 23.8 \\
Tercero & 444 & 16.5 \\
\hline Total & 2.691 & 100.0 \\
\hline
\end{tabular}

TABLA 12 NUMERO DE CONTROLES PRENATALES

\begin{tabular}{|c|r|r|}
\hline Controles & No. & \multicolumn{1}{c|}{$\%$} \\
\hline $1-3$ & 722 & 49.9 \\
$4-6$ & 620 & 42.9 \\
$7-9$ & 82 & 5.7 \\
10 & 22 & 1.5 \\
\hline Total & 1.446 & 100.0 \\
\hline
\end{tabular}


TABLA 13

HABITO DE FUMAR

\begin{tabular}{|c|c|c|c|}
\hline \multicolumn{2}{|c|}{} & No. & $\%$ \\
\hline \multicolumn{2}{|c|}{ NO FUMADORAS } & 1.839 & 70.2 \\
\hline F & $1-5$ & 400 & 15.3 \\
u & $6-10$ & 205 & 7.8 \\
a & $11-15$ & 25 & 1.0 \\
d & $16->$ & 149 & 5.7 \\
r & 16 & & \\
a & & 2.618 & 100.0 \\
\hline
\end{tabular}

TABLA 14

DISTRIBUCION DE LAS PATOLOGIAS

MATERNAS

\begin{tabular}{|c|c|c|c|}
\hline & & No. & $\%$ \\
\hline \multicolumn{2}{|c|}{ SIN PATOLOGIA } & 2.866 & 84.2 \\
\hline \multirow{8}{*}{$\begin{array}{l}\mathbf{P} \\
\mathbf{A} \\
\mathbf{T} \\
\mathbf{O} \\
\mathbf{L} \\
\mathbf{O} \\
\mathbf{G} \\
\mathbf{I} \\
\mathbf{A}\end{array}$} & Otra & 212 & 6.2 \\
\hline & Hipertensión & & \\
\hline & Inducida por & & \\
\hline & el Embarazo & 143 & 4.2 \\
\hline & Amenaza Parto & 138 & 4.0 \\
\hline & Prematuro & & \\
\hline & Hemorragia & 45 & 1.4 \\
\hline & Total & 3.404 & 100.0 \\
\hline
\end{tabular}

TABLA 15

DISTRIBUCION PORCENTUAL DE LA MORBILIDAD MATERNA

\begin{tabular}{|l|r|r|}
\hline PATOLOGIA & No. & \% \\
\hline Otra & 212 & 39.4 \\
Hipertensión Inducida & 143 & 26.6 \\
por el Embarazo & & \\
A. Parto Prematuro & 138 & 25.6 \\
Hemorragia & 45 & 8.4 \\
\hline Total & 538 & 100.0 \\
\hline
\end{tabular}

TABLA 16

TIPO DE HEMORRAGIA SEGUN LA EDAD MATERNA

\begin{tabular}{|l|c|c|c|c|c|c|c|}
\cline { 2 - 8 } \multicolumn{1}{c|}{} & \multicolumn{7}{c|}{ EDAD EN AÑOS } \\
\cline { 2 - 8 } CAUSA & $15-19$ & $20-24$ & $25-29$ & $30-34$ & $35-39$ & $40-44$ & Total \\
\hline Previa & 0 & 7 & 5 & 6 & 2 & 1 & 21 \\
Desprendimiento & 2 & 8 & 5 & 2 & 3 & 4 & 24 \\
\hline Total & 2 & 15 & 10 & 8 & 5 & 5 & 45 \\
\hline
\end{tabular}


TABLA 17

TIPO DE HEMORRAGIA SEGUN LA PARIDAD

\begin{tabular}{|l|r|r|r|r|c|}
\cline { 2 - 6 } \multicolumn{1}{c|}{} & \multicolumn{5}{c|}{ P ARIDAD } \\
\cline { 2 - 6 } \multicolumn{1}{c|}{ CAUSA } & 0 & $1-3$ & $4-6$ & 7 & Total \\
\hline Previa & 2 & 13 & 4 & 2 & 21 \\
Desprendimiento & 7 & 9 & 4 & 4 & 24 \\
\hline Total & 9 & 22 & 8 & 6 & 45 \\
\hline
\end{tabular}

TABLA 18

DATOS DEL PARTO

\begin{tabular}{|l|r|r|}
\cline { 2 - 3 } \multicolumn{1}{c|}{} & \multicolumn{2}{c|}{ TERMINACION DEL PARTO } \\
\cline { 2 - 3 } \multicolumn{1}{c|}{ TIPO PARTO } & No. & $\%$ \\
\hline Espontáneo & 2.498 & 73.5 \\
Fórceps Bajo & 139 & 4.1 \\
Fórceps Medio & 18 & 0.5 \\
Cesárea Electiva & 221 & 6.5 \\
Cesárea Intraparto & 481 & 14.2 \\
Asistencia Pelviana & 25 & 0.7 \\
Extracción Pelviana & 8 & 0.3 \\
Otros & 6 & 0.2 \\
\hline Total & 3.396 & 100.0 \\
\hline
\end{tabular}

TABLA 19

TERMINACION DEL PARTO SEGUN EL ESTADO AL NACER

\begin{tabular}{|l|r|r|r|r|r|}
\cline { 2 - 6 } \multicolumn{1}{c|}{} & \multicolumn{2}{c|}{ VIVO } & \multicolumn{2}{c|}{ MUERTO } & \multicolumn{1}{c|}{} \\
\cline { 2 - 6 } \multicolumn{1}{c|}{ TIPO PARTO } & \multicolumn{1}{c|}{ No. } & $\%$ & \multicolumn{1}{c|}{ No. } & $\%$ & TOTAL \\
\hline Espontáneo & 2.452 & 98.3 & 42 & 1.7 & 2.494 \\
Fórceps Bajo & 138 & 99.3 & 1 & 0.7 & 139 \\
Fórcens Medio & 17 & 94.5 & 1 & 5.5 & 18 \\
Cesárea Electiva & 217 & 98.2 & 4 & 1.8 & 221 \\
Cesárea Intraparto & 465 & 96.9 & 15 & 3.1 & 480 \\
Asistencia Pelviana & 20 & 80.0 & 5 & 20.0 & 25 \\
Extracción Pelviana & 4 & 50.0 & 4 & 50.0 & 8 \\
Oúros & 4 & 66.7 & 2 & 33.3 & 6 \\
\hline Total & 3.317 & 97.8 & 74 & 2.2 & 3.391 \\
\hline
\end{tabular}


TABLA 20

TERMINACION DEL PARTO SEGUN EL PESO AL NACER

\begin{tabular}{|l|r|c|r|r|r|}
\cline { 2 - 6 } \multicolumn{1}{c|}{} & \multicolumn{5}{c|}{ PESO } \\
\cline { 2 - 6 } \multicolumn{1}{c|}{} & \multicolumn{2}{c|}{2.500 gms. } & \multicolumn{2}{c|}{$>2.500$ gms. } & \\
\cline { 2 - 6 } TIPO PARTO & No. & $\%$ & \multicolumn{1}{c|}{ No. } & \% & Total \\
\hline Espontáneo & 303 & 12.2 & 2.175 & 87.8 & 2.478 \\
Fórceps Bajo & 16 & 11.6 & 122 & 88.4 & 138 \\
Fórceps Medio & 2 & 11.1 & 16 & 88.9 & 18 \\
Cesárea Electiva & 38 & 17.5 & 179 & 82.5 & 217 \\
Cesárea Intraparto & 65 & 15.2 & 361 & 84.7 & 426 \\
Asistencia Pelviana & 9 & 39.1 & 14 & 60.9 & 23 \\
Extracción Pelviana & 5 & 62.5 & 3 & 37.5 & 8 \\
Otros & 2 & 33.3 & 4 & 66.7 & 6 \\
\hline Total & 440 & 13.2 & 2.874 & 86.8 & 3.314 \\
\hline
\end{tabular}

TABLA 21

FORMA DE COMIENZO DEL PARTO

INCLUYENDO LOS FETOS MUERTOS

\begin{tabular}{|l|c|c|}
\hline & No. & $\%$ \\
\hline Espontáneo & 3.143 & 99.0 \\
\hline Inducido & 31 & 1.0 \\
\hline Total & 3.174 & 100.0 \\
\hline
\end{tabular}

TABLA 22

ADMINISTRACION DE OXITOCINA DURANTE EL PARTO

\begin{tabular}{|l|r|r|}
\hline & No. & $\%$ \\
\hline Sin Oxitocina & 3.143 & 99.0 \\
Oxitocina P. Dilatante & 21 & 0.7 \\
$\begin{array}{l}\text { Oxitocina P. Dilatante } \\
\text { Más Expulsivo }\end{array}$ & 10 & 0.3 \\
\hline Total & 3.174 & 100.0 \\
\hline
\end{tabular}

TABLA 23

ANESTESIA DURANTE EL PARTO ISE INCLUYEN LOS CASOS DE FETOS MUERTOS)

\begin{tabular}{|l|r|r|}
\hline & No. & \% \\
\hline Sin Anestesia & 1.392 & 41.0 \\
Perineal & 1.075 & 31.6 \\
Peridural & 532 & 15.7 \\
General & 387 & 11.4 \\
Raquídea & 3 & 0.1 \\
Otras & 7 & 0.2 \\
\hline Total & 3.396 & 100.0 \\
\hline
\end{tabular}

TABLA 24

DURACION TOTAL DEL PARTO DESDE 4 - 5 CM. DE DILATACION

\begin{tabular}{|c|r|r|}
\hline & No. & $\%$ \\
\hline Menos de una Hora & 93 & 14.0 \\
$1 \cdot 3$ & 234 & 35.3 \\
$4-6$ & 197 & 29.7 \\
$7-9$ & 138 & 21.0 \\
\hline Total & 662 & 100.0 \\
\hline
\end{tabular}


TABLA 25

TIEMPO TRANSCURRIDO ENTRE LA RUPTURA DE LAS MEMBRANAS Y EL NACIMIENTO

\begin{tabular}{|l|r|r|}
\hline & No. & \multicolumn{1}{c|}{$\%$} \\
\hline Menos de 5 Horas & 2.680 & 85.4 \\
Entre 5 y 23 Horas & 362 & 11.5 \\
24 ó más & 97 & 3.1 \\
\hline Total & 3.139 & 100.0 \\
\hline
\end{tabular}

TABLA 26

INCIDE'JCIA DE PATOLOGIA PUERPERAL

\begin{tabular}{|l|r|r|}
\hline & No. & \multicolumn{1}{|c|}{$\%$} \\
\hline Normales & 2.998 & 88.2 \\
Infección & 396 & 11.6 \\
Hemorragia & 6 & 0.2 \\
\hline Total & 3.400 & 100.0 \\
\hline
\end{tabular}

DATOS DEL RECIEN NACIDO

TABLA 28

TABLA 27

DISTRIBUCION DEL PESO AL NACER

ESTADO DE LA MADRE A LA SALIDA

\begin{tabular}{|l|r|r|}
\hline & No. & \multicolumn{1}{|c|}{$\%$} \\
\hline Sanas & 3.395 & 99.7 \\
Muertas & 9 & 0.3 \\
\hline Total & 3.404 & 100.0 \\
\hline
\end{tabular}

\begin{tabular}{|c|r|r|}
\hline GRAMOS & No. & \multicolumn{1}{|c|}{$\%$} \\
\hline $500-999$ & 18 & 0.5 \\
$1.000-1.499$ & 27 & 0.8 \\
$1.500-1.999$ & 65 & 1.9 \\
$2.000-2.499$ & 330 & 9.8 \\
$2.500-2.999$ & 1.039 & 30.9 \\
$3.000-3.499$ & 1.303 & 38.7 \\
$3.500-3.999$ & 496 & 14.7 \\
$4.000-4.499$ & 87 & 2.7 \\
\hline Total & 3.365 & 100.0 \\
\hline
\end{tabular}

TABLA 29

DISTRIBUCION DEL PESO AL NACER SEGUN LA PARIDAD

\begin{tabular}{|c|c|c|c|c|c|c|c|c|c|}
\hline \multirow[b]{2}{*}{ Peso en Gramos } & \multicolumn{2}{|r|}{0} & \multicolumn{2}{|c|}{$1 \cdot 3$} & \multicolumn{2}{|c|}{$4 \cdot 6$} & \multicolumn{2}{|c|}{$7>$} & \multirow[b]{2}{*}{ Total } \\
\hline & No. & $\%$ & No. & $\%$ & No & $\%$ & No & $\%$ & \\
\hline $500-999$ & 7 & 0.6 & 9 & 0.6 & 2 & 0.5 & 0 & - & 18 \\
\hline $1.000-1.499$ & 10 & 0.8 & 13 & 0.8 & 4 & 1.0 & 0 & - & 27 \\
\hline $1.500-1.999$ & 17 & 1.4 & 35 & 2.1 & 11 & 2.8 & 2 & 1.6 & 65 \\
\hline $2.000-2.499$ & 138 & 11.7 & 145 & 8.7 & 36 & 9.3 & 11 & 8.6 & 330 \\
\hline $2.500-2.999$ & 438 & 37.1 & 486 & 29.1 & 91 & 23.4 & 28 & 21.9 & 1.039 \\
\hline $3.000-3.499$ & 421 & 35.7 & 673 & 40.3 & 157 & 40.5 & 52 & 40.6 & 1.303 \\
\hline $3.500-3.999$ & 137 & 11.6 & 262 & 15.7 & 70 & 18.0 & 27 & 21.1 & 496 \\
\hline $4.000-4.499$ & 16 & 1.1 & 46 & 2.7 & 17 & 4.4 & 8 & 6.2 & 87 \\
\hline Total & 1.184 & 100.0 & 1.669 & 100.0 & 388 & 100.0 & 128 & 100.0 & 3.365 \\
\hline
\end{tabular}


TABLA 30

DISTRIBUCION DEL PESO AL NACER SEGUN

EL INTERVALO INTERGENESICO

\begin{tabular}{|c|r|r|r|r|r|}
\cline { 2 - 6 } \multicolumn{1}{c|}{} & \multicolumn{2}{c|}{$<2.500 \mathrm{gm}}$. & \multicolumn{2}{c|}{$>2.500 \mathrm{gm}}$. & \\
\cline { 2 - 6 } ANNOS & No. & $\%$ & No. & $\%$ & Total \\
\hline 0 & 317 & 76.2 & 781 & 50.8 & 1.098 \\
$1-3$ & 51 & 12.2 & 422 & 27.4 & 473 \\
$4-6$ & 26 & 6.2 & 221 & 14.4 & 247 \\
$>6$ & 22 & 5.4 & 114 & 7.4 & 136 \\
\hline Total & 416 & 100.0 & 1.538 & 100.0 & 1.954 \\
\hline
\end{tabular}

TABLA 31

PUNTAJE DE APGAR

\begin{tabular}{|r|r|r|r|r|}
\cline { 2 - 5 } \multicolumn{1}{c|}{} & \multicolumn{2}{c|}{ Primer Minuto } & \multicolumn{2}{c|}{ Quinto Minuto } \\
\cline { 2 - 5 } \multicolumn{1}{c|}{} & No. & $\%$ & No. & $\%$ \\
\hline 0 & 87 & 2.6 & 79 & 2.4 \\
1.3 & 126 & 3.8 & 19 & 0.6 \\
$4 \cdot 6$ & 252 & 7.5 & 65 & 1.9 \\
$7 \cdot 8$ & 2.874 & 86.1 & 3.185 & 95.1 \\
\hline Total & 3.339 & 100.0 & 3.348 & 100.0 \\
\hline
\end{tabular}

TABLA 32

PUNTAJE DE APGAR A LOS CINCO MINUTOS SEGUN EL HABITO DE FUMAR

\begin{tabular}{|c|c|c|c|c|c|c|c|c|c|c|}
\hline \multirow[b]{3}{*}{ APGAR } & \multicolumn{10}{|c|}{ No. DE CIGARRILLOS } \\
\hline & \multicolumn{2}{|r|}{0} & \multicolumn{2}{|c|}{$1 \cdot 5$} & \multicolumn{2}{|c|}{$6 \cdot 10$} & \multicolumn{2}{|c|}{$11 \cdot 15$} & \multicolumn{2}{|c|}{$16>$} \\
\hline & No. & $\%$ & No. & $\%$ & No. & $\%$ & No. & $\%$ & No. & $\%$ \\
\hline 0 & 36 & 2.0 & 6 & 1.5 & 9 & 4.5 & 1 & 4.4 & 6 & 4.1 \\
\hline $1 \cdot 3$ & 11 & 0.6 & 2 & 0.5 & 0 & - & 0 & - & 1 & 0.6 \\
\hline $4 \cdot 6$ & 42 & 2.4 & 7 & 1.8 & 3 & 1.5 & 0 & - & 2 & 1.4 \\
\hline $7 \cdot 9$ & 1.722 & 95.0 & 378 & 96.2 & 189 & 94.0 & 22 & 95.6 & 138 & 93.9 \\
\hline Total & 1.811 & 100.0 & 393 & 100.0 & 201 & 100.0 & 23 & 100.0 & 147 & 100.0 \\
\hline
\end{tabular}

TABLA 33

APGAR A LOS CINCO MINUTOS SEGUN LA TERMINACION DEL PARTO

\begin{tabular}{|c|r|r|r|r|r|r|r|r|r|r|r|r|r|}
\cline { 2 - 15 } \multicolumn{1}{c|}{} & \multicolumn{2}{c|}{ Espontáneo } & \multicolumn{2}{c|}{ F. Bajo } & \multicolumn{2}{c|}{ Medio } & \multicolumn{2}{c|}{ Cesárea } & \multicolumn{2}{c|}{ Asistencia } & \multicolumn{2}{c|}{ Extracción } & \multicolumn{1}{c|}{ \% } \\
\hline APGAR & No. & \% & No. & $\%$ & No. & $\%$ & No. & $\%$ & No. & $\%$ & No & $\%$ & Total \\
\hline 0 & 45 & 1.8 & 1 & 0.7 & 1 & 5.6 & 21 & 3.0 & 5 & 20.0 & 4 & 50.0 & 77 \\
$1-3$ & 7 & 0.4 & 1 & 0.7 & 0 & - & 9 & 1.3 & 1 & 4.0 & 1 & 12.5 & 19 \\
$4-6$ & 26 & 1.0 & 8 & 5.8 & 0 & - & 29 & 4.2 & 1 & 4.0 & 1 & 12.5 & 65 \\
$7-9$ & 2.376 & 96.8 & 129 & 92.8 & 17 & 94.4 & 634 & 91.5 & 18 & $72.0^{\circ}$ & 2 & 25.0 & 3.176 \\
\hline Total & 2.454 & 100.0 & 139 & 100.0 & 18 & 100.0 & 693 & 100.0 & 25 & 100.0 & 8 & 100.0 & 3.337 \\
\hline
\end{tabular}


TABLA 34

EDAD GESTACIONAL POR EL EXAMEN FISICO

\begin{tabular}{|l|r|r|}
\hline SEMANAS & No. & \% \\
\hline $20-28$ & 13 & 0.5 \\
$29-36$ & 267 & 8.8 \\
$37-42$ & 2.722 & 90.1 \\
$>42$ & 19 & 0.6 \\
\hline Total & 3.021 & 100.0 \\
\hline
\end{tabular}

DISTRIBUCION DEL PESO SEgUN EL ESTADO AL NACER

\begin{tabular}{|r|r|r|r|r|r|}
\cline { 2 - 5 } \multicolumn{1}{c|}{} & \multicolumn{2}{c|}{ VIVOS } & \multicolumn{2}{c|}{ MUERTOS } & \multicolumn{1}{c|}{} \\
\cline { 2 - 5 } \multicolumn{1}{c|}{ GRAMOS } & No. & $\%$ \% & No. & $\%$ & Total \\
\hline $500-999$ & 10 & 58.8 & 7 & 41.2 & 17 \\
$1.000-1.499$ & 17 & 65.4 & 9 & 34.6 & 26 \\
$1.500-1.999$ & 54 & 81.8 & 12 & 8.2 & 66 \\
$2.000-2.499$ & 319 & 96.7 & 11 & 3.3 & 330 \\
$2.500-2.999$ & 1.011 & 99.0 & 10 & 1.0 & 1.021 \\
$3.000-3.499$ & 1.289 & 98.9 & 14 & 1.1 & 1.303 \\
$3.500-3.999$ & 504 & 99.4 & 3 & 0.6 & 507 \\
$4.000-4.499$ & 87 & 100.0 & 0 & 0 & 87 \\
\hline Total & 3.291 & & 66 & & 3.357 \\
\hline \% & 98.0 & & 2.0 & & 100.0 \\
\hline
\end{tabular}

\section{BIBLIOGRAFIA}

1. CENTRO LATINOAMERICANO DE PE. RINATOLOGIA. Incidencia del bajo peso al nacer y mortalidad perinatal en América Latina. Salud Perinatal 1 (1): 3-6, 1983.

2. DIAZ, A. Gonzalo et al. Diagnóstico de situación perinatal. Resultados obtenidos con la aplicación de la historia clínica perinatal en el hospital Sardá de Buenos Aires. Publicación Científica No. 846 del CLAP_OPS/OMS, noviembre 1979.

3. FAUNDES, Anibal et al. Association of marital status and years of schooling with perinatal outcome: The influence of prenatal care as an intermediate variable J. Perinat. Med. 10(2): 105-113, 1982. 
4. GAVIRIA GAVIRIA, Benicio et al. Letalidad perinatal. Rev. Colomb. Obstet. Ginecol. 11(3) 339-350, 1960.

5. JUBIZ HASBUN, Alfonso et al. Diagnóstico de situación perinatal. Rev. Co. lomb. Obstet. Ginecol. 34(1): 51-68, 1983.
6. OCHOA MEJIA, Germán. Mortalidad perinatal. Tesis de Grado. Ant. Médica. 8(1): 2-53, 1958.

7. Idem et al. Resultado perinatal. Informe preliminar. Mimeografiado. Escuela $\mathrm{Na}$ cional de Salud Pública, Medellín. 1976, $8 p$. 